\title{
Growth and Fabrication of GaN/InGaN Violet Light Emitting Diode on Patterned Sapphire Substrate
}

\author{
Sonachand Adhikari1,2, Saroj Kanta Patra1,2, Ashok Lunia1, Sandeep Kumar1, \\ Priyavart Parjapat ${ }^{1}$, Bhoopendra Kushwaha1, Pawan Kumar ${ }^{1}$, Sumitra Singh', \\ Ashok Chauhan', Kuldip Singh", Suchandan Pal1,2, C. Dhanavantri, ${ }^{1,2}$ \\ ${ }^{1}$ CSIR-Network of Institutes for Solar Energy (CSIR-NISE), CSIR-Central Electronics Engineering Research \\ Institute (CSIR-CEERI), Pilani, Rajasthan, India \\ ${ }^{2}$ Academy of Scientific and Innovative Research (AcSIR), Chennai, Tamil Nadu, India \\ Email: sonachand@ceeri.ernet.in
}

Received August 2014

\begin{abstract}
GaN/InGaN based violet light emitting diodes (LEDs), emitting at $430 \mathrm{~nm}$, have been grown on conventional single side polished (SSP) and patterned sapphire substrates (PSS). Characteristics of the epitaxial wafers and subsequently fabricated LEDs have been analyzed. The photoluminescence (PL) peaks have been observed at $428.1 \mathrm{~nm} 426.1 \mathrm{~nm}$ for the epitaxial layers on SSP and PSS respectively. The PL intensity is 2.9 times higher in the case of PSS. The electroluminescence (EL) peaks have been observed at $430.78 \mathrm{~nm}$ and $430.35 \mathrm{~nm}$ for the LEDs on SSP and PSS respectively. The light output from LED fabricated on the PSS is 2.15 times higher than that of the LED on SSP at a forward current of $100 \mathrm{~mA}$.
\end{abstract}

Keywords

LED, GaN/InGaN, PSS

\section{Introduction}

GaN is one of the most widely used direct band gap compound semiconductors with many optoelectronic applications. GaN and its related alloys are currently used extensively for the fabrication of light emitting diodes (LEDs) and laser diodes (LDs). However, the main drawback for this material is the presence of a high threading dislocation density (TDD) due to the lattice mismatch between the sapphire substrate and the epitaxial GaN layer. Growth of epitaxial GaN on free-standing GaN substrate would reduce the TDD; however, the GaN substrates are much costly compared to the sapphire substrates. Another approach to alleviate this problem is the growth of GaN by epitaxial-lateral overgrowth (ELOG) technique, which involves several steps of processing the GaN wafer before the overgrowth. Growth of GaN on patterned sapphire substrate (PSS) is the simplest and most cost effective method to reduce the TDD. In recent years, there has been a tremendous effort to increase 
the performance of nitride based LEDs. One of the methods to improve the light output from LEDs is through the growth of LEDs' epitaxial structure on PSS [1]-[6]. Epitaxial structures grown on PSS also have the added advantages of enhancement in the light extraction from LEDs by providing different escape angles to the light, which otherwise would have been trapped in the GaN LED.

We investigated the performance of LEDs grown by metal-organic chemical vapour deposition (MOCVD) on single side polished (SSP) sapphire substrate and patterned sapphire substrate (PSS). The morphology of the PSS was analyzed using atomic force microscopy (AFM) and optical microscopy. The epitaxial layers were characterized by $x$-ray diffraction (XRD) in a PANalytical X'Pert MRD Pro and photoluminescence (PL) measurement was conducted in Accent RPM2000. Subsequently, violet GaN/InGaN based LEDs (emitting at the wavelength of $430 \mathrm{~nm}$ ) have been fabricated and their electrical and optical properties have been analyzed.

\section{Experimental Methods}

\subsection{Growth of Epitaxial Structure}

The epitaxial structure of the LED was grown on the SSP and PSS simultaneously in a Thomas Swan $3 \times 2$ CCS MOCVD. $\mathrm{NH}_{3}$ was used as the source of $\mathrm{N}$; TMGa, TMIn, TMAl and $\mathrm{Cp}_{2} \mathrm{Mg}$ were used as the sources of $\mathrm{Ga}$, In, $\mathrm{Al}$ and $\mathrm{Mg}$ respectively. $1 \% \mathrm{SiH}_{4}$ in hydrogen was used for the $n$-type doping. The structure consists of $30 \mathrm{~nm}$ thick nucleation layer, $3 \mu \mathrm{m}$ thick undoped $\mathrm{GaN}$ followed by $1 \mu \mathrm{m}$ thick n-type $\mathrm{GaN}$ with a carrier concentration of $\sim 6 \times 10^{18} \mathrm{~cm}^{-3}$. The active region consists of $3 \mathrm{~nm}$ thick five quantum wells of InGaN separated by $10 \mathrm{~nm}$ thick GaN barriers. $15 \mathrm{~nm}$ thick $\mathrm{Al}_{0.15} \mathrm{Ga}_{0.85} \mathrm{~N}$ was employed as an electron blocking layer followed by a $120 \mathrm{~nm}$ thick $p$-GaN layer with a carrier concentration of $\sim 5 \times 10^{17} \mathrm{~cm}^{-3}$.

\subsection{Fabrication of LED}

LEDs were fabricated from the epitaxial structures grown on SSP and PSS. The fabrication steps involve patterning by lithography and subjecting to $\mathrm{Cl}_{2} / \mathrm{BCl}_{3}$ reactive ion etching to a depth of $600 \mathrm{~nm}$ to form a mesa. Pre-metallization etch for the $n$-type contact was carried out in buffer-oxide etchant for 1 minute. Ti/Al/Ni/Au $(20 \mathrm{~nm} / 150 \mathrm{~nm} / 40 \mathrm{~nm} / 50 \mathrm{~nm}$ ) was deposited using electron beam evaporation followed by lift-off and rapid thermal annealing at $850^{\circ} \mathrm{C}$ for $30 \mathrm{sec}$ to form Ohmic contact to the n-type GaN. Pt/Ni/Au (3 nm/3 nm/4 nm) was deposited and rapid thermal annealed at $550^{\circ} \mathrm{C}$ for $300 \mathrm{sec}$ in $\mathrm{N}_{2}$ ambient to form the transparent current spreading layer. $\mathrm{Ni} / \mathrm{Au}(20 \mathrm{~nm} / 100 \mathrm{~nm})$ were deposited upon the current spreading layer and annealed at $550^{\circ} \mathrm{C}$ for 300 sec to form the contacts to $p$-GaN.

\section{Results and Discussion}

The morphology of the PSS was analysed by AFM and optical microscopy. The PSS consists of conical protrusions with a pattern height of $\sim 500 \mathrm{~nm}$, a periodicity of $3 \mu \mathrm{m}$ having a basal diameter of $2 \mu \mathrm{m}$. The AFM and optical images of the PSS is shown in Figure 1.

XRD measurement for the epitaxial layers grown on both SSP and PSS were carried out in symmetric (002) and asymmetric (102) planes for the estimation of TDD through broadening of XRD curves. The symetric (002) peak is broadened by screw dislocation $N_{\text {screw }}$, which can be calculated as suggested by [7]

$$
N_{\text {screw }}=\left(\mathrm{FWHM}_{(002)}\right)^{2} /\left(9 b_{\text {screw }}\right)^{2}
$$

where $b_{\text {screw }}$ is the Burgers vector corresponding to screw dislocation $\left(b_{\text {screw }}=0.5185 \mathrm{~nm}\right)$. Edge dislocation density $N_{\text {edge }}$ have been estimated from the asymmetric (102) scan of of the epitaxial layers. The $N_{\text {edge }}$ has been calculated as

$$
N_{\text {edge }}=\left(\operatorname{FWHM}_{(102)}\right)^{2} /\left(9 b_{\text {edge }}\right)^{2}
$$

where $b_{\text {edge }}$ is the Burgers vector corresponding to edge dislocation $\left(b_{\text {edge }}=0.3189 \mathrm{~nm}\right)$. Therefore, the total dislocation density $N_{\text {total }}$ can be calculated as

$$
N_{\text {total }}=N_{\text {screw }}+N_{\text {edge }}
$$

The above calculations are summarized in Table 1. The $N_{\text {total }}$ of the PSS $\left(6.25 \times 10^{8} \mathrm{~cm}^{-2}\right)$ is lower than that 
of SSP $\left(7.92 \times 10^{8} \mathrm{~cm}^{-2}\right)$.

The photoluminescence (PL) measurement of the LED structure on both SSP and PSS were carried out and their PL emission patterns are shown in Figure 2. The peak wavelengths of SSP and PSS epitaxial LED structures were observed at $428.1 \mathrm{~nm}$ and $426.1 \mathrm{~nm}$ respectively. The PL intensity from the PSS is 2.9 times compared to that of the SSP under the same measurement conditions, which qualitatively indicates a much better epitaxial layer structure in the case of PSS.

Electroluminescence (EL) measurements were carried out after the fabrication of LEDs at a current of 100 $\mathrm{mA}$. The EL spectra for the LEDs fabricated on SSP and PSS substrates is shown in Figure 3. The EL spectra closely follows their respective PL spectra. EL intensity of the LED on PSS is 2.7 times higher compared to that on the SSP, which is due to the enahncement in extration of light because of the underlying PSS.

Specific Contact resistance of $\mathrm{Ti} / \mathrm{Al} / \mathrm{Ni} / \mathrm{Au}$ on $n-\mathrm{GaN}$ and $\mathrm{Ni} / \mathrm{Au}$ on $p$-GaN were measured through the transfer length method (TLM) for both SSP and PSS samples and are summarized in Table 2. The resistance of the samples were obtained by sweeping the voltage from -2 to $+2 \mathrm{~V}$ and measuring the current. I-V characteristics were linear, which meant that the contacts to both $n$-GaN and $p$-GaN were Ohmic in nature. The contact resistance was of the order of few $\Omega$ s for $n-G a N$ and few $\mathrm{k} \Omega \mathrm{s}$ for $p$-GaN layer respectively.

I-V and L-I characteristics of the fabricated LED samples on SSP and PSS are plotted in Figure 4. It is evident that the LEDs fabricated on PSS have higher light output power as compared to those of the LEDs fabricated on SSP. At a current of $100 \mathrm{~mA}$, the light output from the LEDs on PSS was 2.15 times higher than the LEDs on SSP. The above result is due to the fact that micro-pattering of sapphire substrate provides different escape angles to light which results in higher light extraction efficiency.

\section{Conclusion}

Violet emitting GaN/InGaN based epitaxial structures of LED have been grown in MOCVD on SSP and PSS. The TDDs on the SSP and PSS have been evaluated by XRD and it is observed that the TDDs have been reduced in the epitaxial layers grown on PSS. The PL emission intensity of the LED structure on PSS is 2.9 timeshigher compared to the one on SSP, which infers better epitaxial layer on the PSS. The EL and L-I characteris-

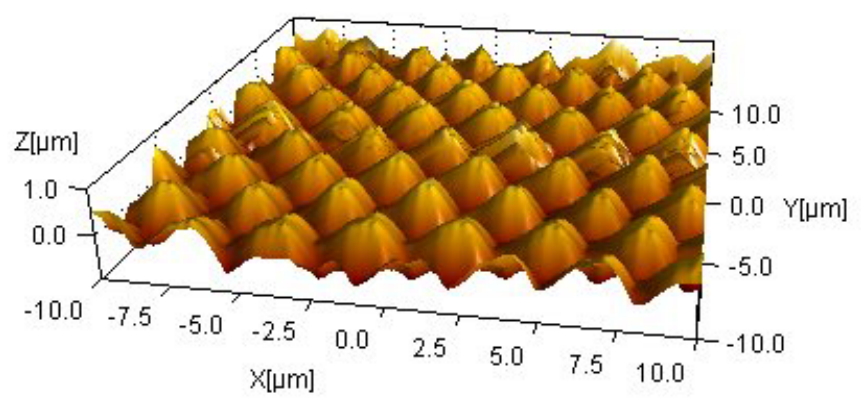

(a)

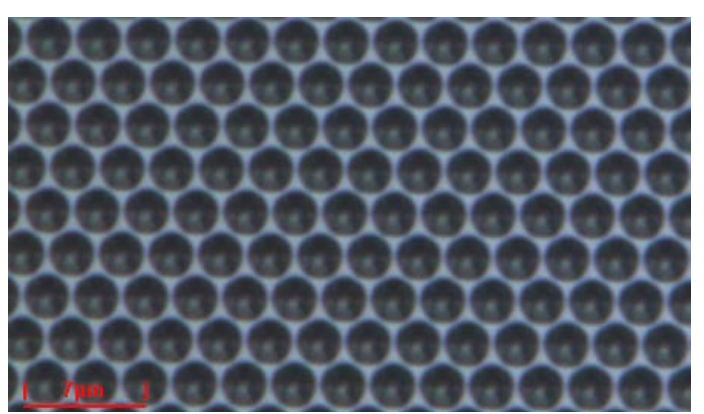

(b)

Figure 1. (a) AFM image of the PSS; (b) Optical microscopy image of the PSS.

Table 1. Summary of the $N_{\text {screw }}, N_{\text {edge }}$ and $N_{\text {total }}$ determined from the XRD measurement.

\begin{tabular}{cccccc}
\hline \multirow{2}{*}{ Sample } & \multicolumn{2}{c}{ FWHM (arcsec) } & \multicolumn{3}{c}{ Dislocation Density $\left(\mathrm{cm}^{-2}\right)$} \\
\cline { 2 - 6 } & $(002)$ & $(102)$ & $N_{\text {Screw }}$ & $N_{\text {Edge }}$ & $N_{\text {Total }}$ \\
\hline SSP & 256 & 533 & $6.39 \times 10^{7}$ & $7.29 \times 10^{8}$ & $7.92 \times 10^{8}$ \\
PSS & 283 & 462 & $7.76 \times 10^{7}$ & $5.48 \times 10^{8}$ & $6.25 \times 10^{8}$ \\
\hline
\end{tabular}

Table 2. Specific contact resistance measurement on both n-contact and p-contact for SSP and PSS.

\begin{tabular}{ccc}
\hline \multirow{2}{*}{ Sample } & \multicolumn{2}{c}{ Specific contact resistance $\left(\Omega \mathrm{cm}^{2}\right)$} \\
\cline { 2 - 3 } & N-Type & P-Type \\
\hline SSP & $7.84 \times 10^{-5}$ & $1.28 \times 10^{-2}$ \\
PSS & $4.77 \times 10^{-5}$ & $2.89 \times 10^{-2}$ \\
\hline
\end{tabular}




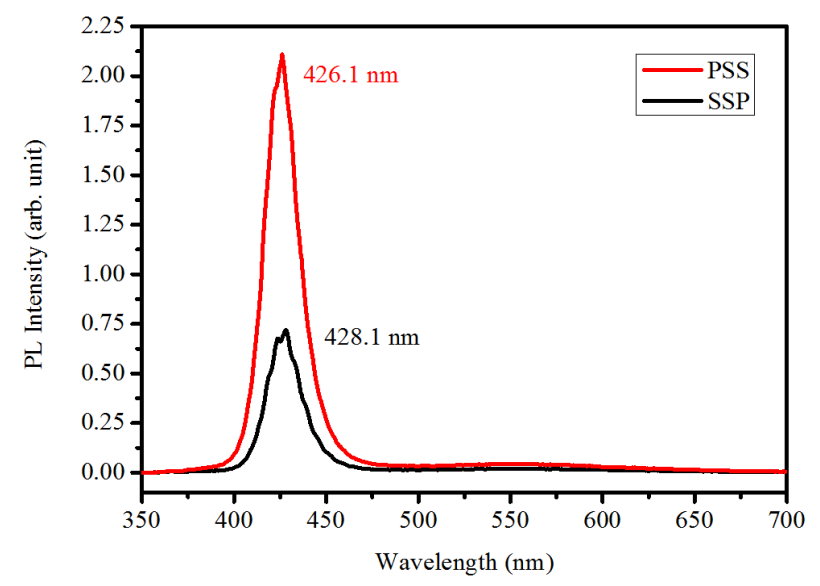

Figure 2. PL spectra of LED epitaxial structures on SSP and PSS.

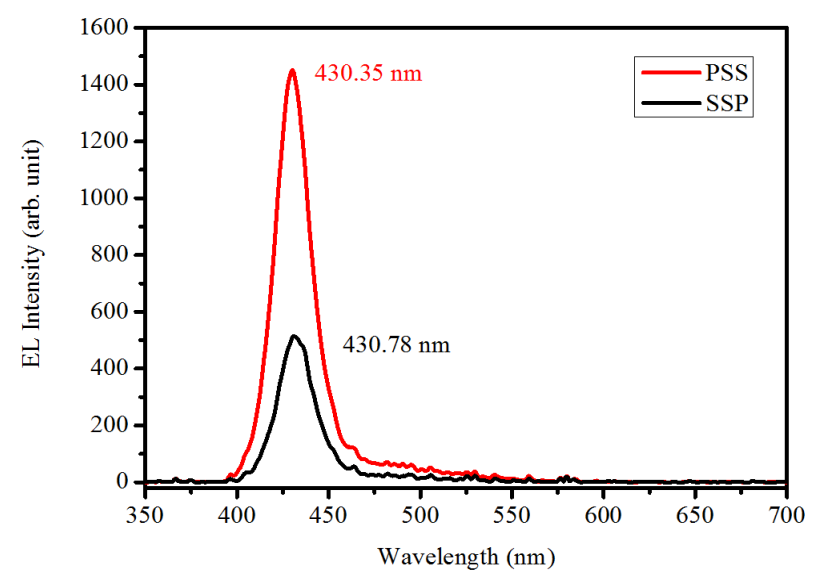

Figure 3. EL spectra of LED epitaxial structures on SSP and PSS.

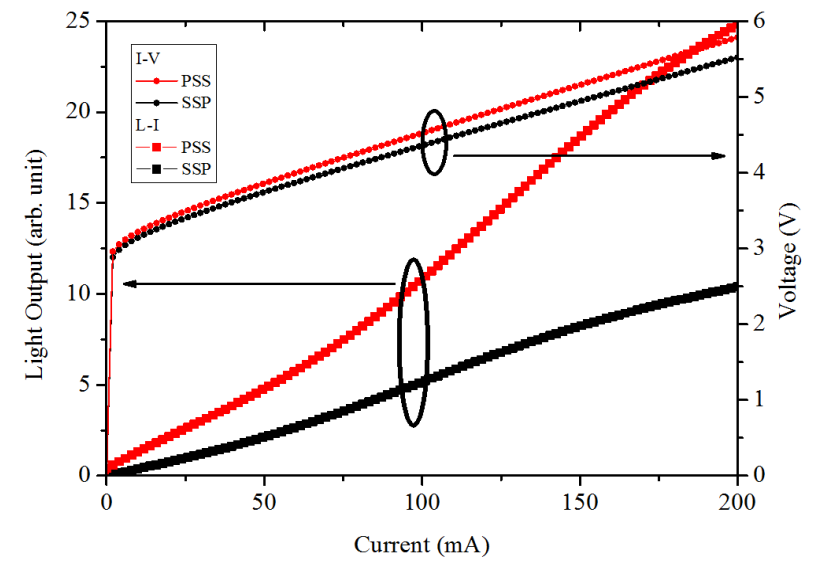

Figure 4. L-I and I-V characteristics of the LEDs on SSP and PSS.

tics confirm the enhancement of light extraction in the case of PSS. The light output at $100 \mathrm{~mA}$ is 2.15 times in the case of PSS.

\section{Acknowledgements}

Authors are thankful to the director, CSIR-CEERI-Pilani for his encouragement and constant support. Authors 
also acknowledge the CSIR for funding TAPSUN programme by sponsoring NWP-55 project.

\section{References}

[1] Tadatomo, K., Okagawa, H., Ohuchi, Y., Tsunekawa, T., Jyouichi, T., Imada, Y., et al. (2001) High Output Power InGaN Ultraviolet Light-Emitting Diodes Fabricated on Patterned Substrates Using Metalorganic Vapor Phase Epitaxy. Physica Status Solidi (a), 188, 121-125. http://dx.doi.org/10.1002/1521-396X(200111)188:1<121::AID-PSSA121>3.0.CO;2-G

[2] Chang, S.J., Lin, Y.C., Su, Y.K., Chang, C.S., Wen, T.C., Shei, S.C., et al. (2003) Nitride-Based LEDs Fabricated on Patterned Sapphire Substrates. Solid-State Electronics, 47, 1539-1542. http://dx.doi.org/10.1016/S0038-1101(03)00073-X

[3] Feng, Z.H., Qi, Y.D., Lu, Z.D. and Lau, K.M. (2004) GaN-Based Blue Light-Emitting Diodes Grown and Fabricated on Patterned Sapphire Substrates by Metalorganic Vapor-Phase Epitaxy. Journal of Crystal Growth, 272, 327-332. http://dx.doi.org/10.1016/j.jcrysgro.2004.08.070

[4] Kao, C.-C., Su, Y.-K., Hsieh, Y.-T., Lee, Y.-C., Cheng, C.-Y. and Lin, C.-L. (2011) Investigation of GaN-Based Light-Emitting Diodes Grown on Patterned Sapphire Substrates by Contact-Transferred and Mask-Embedded Lithography. Applied Physics Express, 4, 062102-062104. http://dx.doi.org/10.1143/APEX.4.062102

[5] Yamada, M., Mitani, T., Narukawa, Y., Shioji, S., Niki, I., Sonobe, S., et al. (2002) InGaN-Based Near-Ultraviolet and Blue-Light-Emitting Diodes with High External Quantum Efficiency Using a Patterned Sapphire Substrate and a Mesh Electrode. Japanese Journal of Applied Physics, 41, L1431-L1433. http://dx.doi.org/10.1143/JJAP.41.L1431

[6] Yu, S.-F., Chang, S.-P., Chang, S.-J., Lin, R.-M., Wu, H.-H. and Hsu, W.-C. (2012) Characteristics of InGaN-Based Light-Emitting Diodes on Patterned Sapphire Substrates with Various Pattern Heights. Journal of Nanomaterials, 2012. http://dx.doi.org/10.1155/2012/346915

[7] Moram, M.A. and Vickers, M.E. (2009) X-Ray Diffraction of III-Nitrides. Reports on Progress in Physics, 72, Article ID: 036502. http://dx.doi.org/10.1088/0034-4885/72/3/036502 